\title{
Melatonin Dependent Modulation in microRNAs Relative Expression Pattern and Levels of cfDNA in Mature Oocytes of Unexplained Infertile Patients.
}

\section{Haroon Latif Khan}

Lahore Institute of Fertility and Endocrinology

Shahzad bhatti ( $\nabla$ drshahzadbhatti@yahoo.com )

Lahore Institute of Fertility and Endocrinology https://orcid.org/0000-0003-1937-0100

\section{Sana abbas}

Lahore Institute of Fertility and Endocrinology

Celal Kaloglu

Cumhuriyet Universitesi

\section{Syeda Qurat-ul-ain}

Kinnaird College for Women

\section{Yousaf Latif Khan}

Lahore Institute of Fertility and Endocrinology

\section{Zahira Hassan}

Royal Free London NHS Foundation Trust

Hikmet Hakan Aydin

Ege Universitesi

Nilgün Öztürk Turhan

Bayindir Hastanesi Sogutozu

Nour El Houda Bousnane

Universite Batna 2

Aysegul Yildiz

Mugla Sitki Kocman Universitesi

Isadora Saruhashi

Universidade Tuiuti do Parana

\section{Research}

Keywords: Melatonin, miRNA expression, Cell-free DNA, follicular fluid, embryo quality, Unexplained infertility

Posted Date: August 6th, 2020 
DOI: https://doi.org/10.21203/rs.3.rs-53356/v1

License: (c) (1) This work is licensed under a Creative Commons Attribution 4.0 International License. Read Full License 


\section{Abstract}

\section{Background}

Intrafollicular melatonin maintains the DNA integrity of granulosa cells and protects them against apoptosis. This ubiquitous indoleamine compound serves as a potent free radical scavenger. It reduces oxidative stress and modulates DNA damage response, which improves oocyte's quality with a higher fertilization rate.

\section{Methods:}

This prospective study was designed to investigate the antioxidant property of intrafollicular melatonin and its impact on IVF outcome parameters by exploring the relative expression of five microRNAs (miR663b, miR-320a, miR-766-3p, miR-132-3p, and miR-16-5p) and levels of cfDNA by real-time PCR in unexplained infertile patients. We collected 425 follicular fluid (ff) samples containing mature oocytes from 295 patients undergoing IVF.

\section{Results:}

Patients were sub-grouped based on intrafollicular melatonin concentration (Group A; $\leq 30 \mathrm{pg} / \mathrm{mL}$, Group $B ;>70$ to $\leq 110 \mathrm{pg} / \mathrm{mL}$ ), Group $\mathrm{C} ;>111$ to $\leq 385 \mathrm{pg} / \mathrm{mL}$ ). Our results showed that patients with $\leq 30$ $\mathrm{pg} / \mathrm{mL}$ intrafollicular melatonin levels have a significantly higher cfDNA levels and lower relative expression of miR-663b, miR-320a, miR-766-3p, miR-132-3p, and miR-16-5p compared to other subgroups $(p<0.001)$. Similarly, they have a low fertilization rate and a reduced number of high-quality day 3 embryos. For the probability of obtaining a good quality embryo, miRNAs had AUC value of 0.89 [ $95 \% \mathrm{Cl}$; $0.71 ; 0.93$ ] with $87 \%$ sensitivity and $83 \%$ specificity $(p=0.001)$, whereas cfDNA have AUC value 0.76 [cutoff points, $95 \% \mathrm{Cl}: 0.61,0.67 ; 0.85]$ along with $82.4 \%$ senstivity and $66.7 \%$ specificity.

\section{Conclusion:}

Conclusively, our study provided evidence that melatonin's antioxidant capability significantly impacts cfDNA concentration and miRNAs relative expression profile in the follicular microenvironment for optimal oocyte development and embryo quality. Therefore, it may be a potent non-invasive diagnostic tool to select high-quality day 3 embryos with such promise.

\section{Background}

Melatonin (N-acetyl-5-methoxytryptamine) is a potent immunomodulatory synthetic product of the pineal gland that manages the circadian rhythm, sleep-wake cycle, blood pressure, and monitors mammalian reproductive activities. Moreover, this ubiquitous indoleamine compound serves as a potent free radical scavenger. Melatonin, in part, functions through the activation of two high-affinity G-protein-coupled 
receptors. However, it was initially uncovered as a pleiotropic regulatory molecule that has shown considerable antioxidant/antinitrosant activity in various cell types and biological fluids, including ff [1]. Intrafollicular melatonin concentration is almost 3-fold higher than that in blood plasma, which directly protects oocyte from oxidative stress within the ovarian follicle [2]. Human follicular fluid (ff) may be considered a "biological window" that reflects allosteric metabolic regulation. It comprises various hormones, polysaccharides, reactive oxygen species (ROS), and antioxidant defense systems [3]. Paradoxically, ROS act as powerful signaling molecules that initiate the process of various growth-related responses and are primarily involved in cellular metabolism. However, the acquisition of ROS has harmful effects on the follicular microenvironment. Surprisingly, it favors the process of ovulation, thus might function as a double-edged-sword in the cellular processes [4]. Under moderate concentrations, ROS are responsible for mediating inter-and intracellular signaling cascades provided protection against apoptosis while massive production of ROS result in oxidative stress. Apoptosis is mainly due to the upregulation of oxidant species and the antioxidant defense system's limited efficacy. However, within the follicles, a delicate equilibrium exists between the pro-oxidant and antioxidant defense system, maintained by antioxidants' prominence. Non-enzymatic antioxidants, such as vitamins, minerals, superoxide dismutase (SOD), Glutathione (GSH), ascorbic acid, and melatonin, are required necessarily during ovulation [5].

Oxidative stress is an incessant cause of DNA damage and epigenetic modifications in developing oocytes. The molecular mechanism of biological processes underlying cellular response to broadspectrum actions of melatonin is not restricted to its interaction with intracellular proteins but also has functional effects on non-protein-coding RNA species [6]. Owing to their wide plethora of functional activities and dynamic stability, miRNAs have a promising function as diagnostic and prognostic biomarkers [7]. Notably, miRNAs expression profile has been recognized as a novel mechanism of intercellular communication, thereby acting similarly to hormones and a potential source of non-invasive biomarkers in the oocyte microenvironment to predict embryo quality [8].

Oxidative or nitro-oxidative stress leads to DNA damage when the antioxidant capability is insufficient, thereby playing a crucial role in intrinsic/extrinsic pathways of apoptosis by the integrated release of various pro-apoptotic factors. The amount of cfDNA in ff significantly related to the oocyte's quality and reflects the extent of cell damage by apoptosis [9]. In contrast, intrafollicular melatonin maintains the DNA integrity of granulosa cells, protects them against apoptosis, and stimulates estradiol and progesterone production that improves oocyte's quality with a higher fertilization rate [10]. Considering the possible effect of melatonin on different miRNA expression levels, which affects oocyte maturation quality, it should be of primary importance to focus on this relationship. Currently, limited studies are focused on the interactions of melatonin concentration and miRNAs relative expression profile in the ff samples, which might serve as a potent non-invasive tool for predicting oocyte development capability in infertile patients.

Therefore, this study was designed to investigate the melatonin-dependent modulation in five miRNAs (miR-663b, miR-320a, miR-766-3p, miR-132-3p, and miR-16-5p) relative expression patterns constitutively 
in mature oocytes' ff microenvironment to predict embryo quality. Previous studies demonstrating their involvement in oocyte maturation and embryo quality [11, 12]. Additionally, we sought to explore whether melatonin's antioxidative property affects the concentration of apoptotic cfDNA in ff samples from which mature oocytes were obtained.

\section{Methods}

\section{Participant's Selection:}

\section{Subjects}

This prospective study included 425 individual ff samples related to mature oocytes from 295 women (mean age; $33.87 \pm 1.98$ years) with unexplained infertility. The subjects were registered in the tertiarycare hospital's assisted reproductive center between January 2017 and December 2018. The participants were sub-grouped based on intrafollicular melatonin concentration (Group $A ; \leq 30 \mathrm{pg} / \mathrm{mL}$, Group $B ;>70$ to $\leq 110 \mathrm{pg} / \mathrm{mL}$ ), Group $\mathrm{C} ;>111$ to $\leq 385 \mathrm{pg} / \mathrm{mL}$ ). The study was approved by the Institutional Review Board (IRB). All patients provided written consent to participate.

\section{Inclusion Criteria}

Unexplained infertile women with less than four previous attempts and have normal physical and mental health, basal FSH level $\leq 8.85 \mathrm{IU} / \mathrm{mL}$, and ovaries appeared in normal shape and sizes, were the part of this study. Moreover, male partners have normal semen parameters.

\section{Exclusion Criteria}

We excluded patients with metabolic disorders, communicable diseases, body mass index (BMI) $\geq 35$ $\mathrm{kg} / \mathrm{m}^{2}$, hyperandrogenemia, polycystic ovary syndrome, hyperprolactinemia, and reported any pelvic surgery. Moreover, we excluded females with known chromosomal translocation, endometriosis, diminished ovarian reserve, and autoimmune diseases.

\section{Assessment of Clinical Parameters}

BMI was calculated based on height and weight. While baseline hormones such as follicular stimulating hormone $(\mathrm{FSH})$, luteinizing hormone $(\mathrm{LH}), 17 \beta$-estradiol $\left(\mathrm{E}_{2}\right)$, thyroid-stimulating hormone $(\mathrm{TSH})$ and antimullerian duct hormone $(\mathrm{AMH})$ were assessed on $2^{\text {nd }}$ day of the menstrual cycle through electrochemiluminescence immunoassay, according to the manufacturer's instructions (Elecsys ${ }^{\circledR}$ Roche Diagnostics, Indianapolis, USA). The antral follicle count (AFC) was assessed using transvaginal ultrasonography (TVS) on the $2^{\text {nd }}$ or $3^{\text {rd }}$ day of the menstrual cycle. 


\section{Therapeutic Regimen}

To minimize the possible confounding bias by varied controlled ovarian stimulation (COS) procedures, we only include patients in which ovarian stimulation was done through long GnRH agonist (decapeptyl ${ }^{\circledR}$ : Ferring, USA, ATCO pharma) administered in the middle of the luteal phase of the previous cycle. Ovarian stimulation with rFSH (Follitropin $\beta$, Purigon ${ }^{\circledR}$ : Organon Schering-plough, Oss, France) was evaluated by TVS and by quantifying serum $17 \beta$-estradiol level. The dosage of $\mathrm{rFSH}$ was adjusted according to ovarian response, BMI, bFSH levels, and AFC. A single dose of human chorionic gonadotrophin (650010,000 IU: Merk Serono, Lyon, Spain) was injected when more than two follicles reached a mean diameter of $18 \mathrm{~mm}$ or more by TVS inspection.

\section{Follicular fluid collection and estimation of melatonin and $E_{2}$ concentration}

Oocytes were retrieved by TVS guided puncture after 36 hours of hCG treatment. Clear follicular fluid without blood contents was aspirated independently from the two or three individual follicles. FF samples of mature MII-oocytes were centrifuged separately at $1500 \times \mathrm{g}$ for 20 minutes at $4{ }^{\circ} \mathrm{C}$ by preventing highintensity bright light exposure. The supernatant was filtered through a $0.85 \mu \mathrm{L}$ filter and stored immediately as aliquots of $500 \mu \mathrm{L} \times 2$ at $-80^{\circ} \mathrm{C}$. Each mature oocyte, its related embryo, and ff sample were handled separately in the IVF laboratory. Subsequently, to avoid potential confounding bias by dissimilar follicles of different maturation statuses, as they might contain a varied miRNA and cfDNA profile, we only include those follicles with greater than $18 \mathrm{~mm}$ diameter. Finally, mature oocytes were subject to intracytoplasmic sperm injection (ICSI) procedure. Melatonin and $\mathrm{E}_{2}$ concentrations were evaluated by diluting ff samples 1:100 through radioimmunoassay kits $(\mathrm{MP} \AA$ diagnostics, Santa Ana, California, USA). Intra-assay variations for melatonin and $\mathrm{E}_{2}$ was $<10 \%$.

\section{Assessment of embryo quality}

Fertilization check was done 18-24 $\mathrm{h}$ after ICSI, and the embryo quality was determined through manual grading, using standard criteria based on cytoplasmic appearance, the extent of fragmentation, number, and regularity in the symmetry of blastomeres [13].

\section{RNA extraction from follicular fluid and relative expression analysis by $R T-q P C R$}

$500 \mu \mathrm{L}$ frozen aliquots were thawed on ice, and cell debris was removed by centrifugation for $20 \mathrm{~min}$ at $3000 \times \mathrm{g}$ at $4{ }^{\circ} \mathrm{C}$. RNA was extracted from individual follicles using the Silica-based membrane purification technique (miRNAeasy kit, Qiagen, USA) following the given instructions except that we diluted the sample 3:1 ratio with XBP buffer to optimize its use with the ff. Total RNA was dissolved in 30 
$\mu \mathrm{L}$ of RNAs free water, and its concentration was measured through Nanodrop ND-1000 spectrophotometer (NanoDrop Technologies, Wilmington, DE, USA). Expression-based digital gel electrophoresis (Bio-Rad, Hercules, CA, USA) was also used to confirm the total RNA concentration. MicroRNA profiling and data normalization were achieved as narrated by (Mestdagh et al 2009). Complementary DNA (cDNA) was generated using the TaqMan MicroRNA reverse transcription kit (Life Technologies, USA) in combination with RNA specific stem-loop Megaplex primers (Applied biosystems). A total of $15 \mu \mathrm{L}$ reaction mixture contains $5 \mu \mathrm{L}$ of a sample (10 $\mathrm{ng}$ miRNA), $0.5 \mu \mathrm{L}$ of dNTP $(100 \mathrm{mM}), 1.5$ $\mu \mathrm{L}$ RT buffer (10X), $1 \mu \mathrm{L} / 50 \mathrm{IU}$ of multiScribe RT enzyme, $0.19 \mu \mathrm{L}$ of RNase inhibitor, $3 \mu \mathrm{L}$ of stem-loop RT primers and $4.16 \mu \mathrm{L}$ of nuclease-free water. Reverse transcription was performed in pulsating RT reaction: 40 cycles of $16^{\circ} \mathrm{C}$ for $2 \mathrm{~min}, 42^{\circ} \mathrm{C}$ for 60 seconds, and $50^{\circ} \mathrm{C}$ for 60 seconds. Inactivation of reverse transcriptase was done at $85^{\circ} \mathrm{C}$ for $5 \mathrm{~min}$ and hold step at $4{ }^{\circ} \mathrm{C}$. Amplification was done with the following conditions: enzyme inactivation at $95^{\circ} \mathrm{C}$ for $10 \mathrm{~min}$ and 40 cycles of two thermal amplification steps of $95^{\circ} \mathrm{C}$ for 15 seconds 60 seconds for $1 \mathrm{~min}$ and a hold step at $4{ }^{\circ} \mathrm{C}$. Q-PCR was duplicated for each sample using a CFX- $96^{\circledR}$ touch RT-PCR detection system (Bio-Rad, Life Sciences, USA). We used Allele ID software ${ }^{\circledR}$ to design primers and probs. For quantification of follicular fluid miRNAs expression levels, PCR reaction was performed in a total volume of $20 \mu \mathrm{L}$, having $3 \mu \mathrm{L}$ of cDNA, $10 \mu \mathrm{L}$ of TaqMan Universal PCR MasterMix (Applied Biosystems), $0.8 \mu \mathrm{L}$ of each primer and $5.4 \mu \mathrm{L}$ double distilled water. Amplification was carried out in a 96-well plate, and thermocycling conditions were 10 minutes at $95^{\circ} \mathrm{C}$ for enzyme activation, followed by 45 cycles of $95^{\circ} \mathrm{C}$ for 20 seconds, $60^{\circ} \mathrm{C}$ for 60 seconds. miRNA expression levels were normalized against the expression of MiR-16, which was used as an internal control because of its constant expression in $\mathrm{ff}$ samples. The relative expression of the five miRNAs such as miR-320a, miR766-3p, miR-132-3p, miR-16-5p, and miR-663b was calculated using equation $2^{-\Delta C t}$, while $\Delta \mathrm{Ct}=\mathrm{Ct}$ target miRNA $-\mathrm{Ct}$ miR-16. To calculate the fold change $(\mathrm{FC})$, we estimated the relative expression levels between high quality and impaired quality embryo on day 3 using $2^{-\Delta \Delta C r t}$ formula [11].

\section{Extraction and assessment of follicular fluid cell-free DNA (cfDNA)}

CfDNA was quantified, as previously described [14]. For cfDNA extraction, each ff sample was diluted with an equal volume of buffer solution (Tween-20, Tris- $50 \mathrm{mmol} / \mathrm{I}$, EDTA-1 mmol) and incubated with proteinase $\mathrm{K}$ (Qiagen) at $55^{\circ} \mathrm{C}$ for at least $30 \mathrm{~min}$, followed by inactivation at $98{ }^{\circ} \mathrm{C}$ for $10 \mathrm{~min}$. After denaturation, each ff sample was centrifuged at $3000 \mathrm{rpm}$ for $15 \mathrm{~min}$ and then immediately stored at -80 ${ }^{\circ} \mathrm{C}$ until quantification. The cfDNA concentration in each follicle with mature oocyte was estimated relative to the corresponding amplification of $\beta$-globin and GAPDH measured by the real-time PCR-SYBR green detection method previously described [15].

\section{Antioxidant status and oxidative stress markers measurements in follicular fluid samples}


The frozen $\left(-80^{\circ} \mathrm{C}\right) \mathrm{ff}$ samples were thawed and evaluated for oxidative status. Average values of triplicate measurements were carried out from each ff sample to avoid inter-assay variations. ROS levels were measured by chemiluminescence assay using luminol (5-amino-2,3-dihydro-1,4-phthalazinedione) as a prob [16]. The total antioxidant capacity (TAC) was assessed using the colorimetric assay based on the manufacturer's instructions (BioVision, Inc, CA, USA). Lipid peroxidation was evaluated by calculating the concentration of Thiobarbituric acid reactive substances (TBARS) [17] while 8-hydroxy-2'deoxyguanosine (8-OHdG) was measured using a kit based on the manufacturer's instructions (BioVision, Inc, CA, USA). Both TBARS and 8-OHdG values were expressed as $\mu \mathrm{M} / \mathrm{L}$ and $\mathrm{ng} / \mathrm{mL}$, respectively.

\section{Pathway analysis:}

We performed in-silico analysis to predict targets of miRNAs using web-based bioinformatics tool DIANA miRPath-v3 available on http://snf-515788.vm.okeanos.grnet.gr. Pathways were identified in both the regression analysis and fold-change. The results were demonstrated as a heat map. The more intense red color directed an increased probability that a specific miRNA targets a unique pathway supplemented with target genes.

\section{Statistical Analysis:}

Baseline characteristics are presented as means \pm SD, number percentage [ $\mathrm{n} \%$, and median with $95 \%$ population limits as applicable. We used Kruskal-Wallis/two-tailed test to explore the outcome differences in parameter levels between patients with low $(\leq 30 \mathrm{pg} / \mathrm{ml})$, intermediate (>70 to $\leq 110$ $\mathrm{pg} / \mathrm{ml})$ ), and high intrafollicular melatonin concentration (>111 to $\leq 385 \mathrm{pg} / \mathrm{ml})$. Based on the evaluation of the normality of the distribution by Kolmogorov-Smirnov test and Shapiro-Wilk test, we used the MannWhitney $U$ test to determine the Pairwise comparison between different groups. $\chi^{2}$-test was used to address the categorical variables. Spearman rank test was used to determine the correlation between intrafollicular melatonin levels and other parameters. Receiving operating characteristics (ROC) curves were used to calculate AUC with a $95 \%$ confidence interval $(\mathrm{Cl})$. The sensitivity and specificity for optimal cut-off were calculated using XLSTAT 2020 software. SPSS (version 27; SPSS Inc., Chicago, IL. USA) was used for further statistical analysis. $P<0.05$ was considered statistically significant.

\section{Results}

\section{Baseline clinical characteristics of participants}

The demographic parameters of participants are listed in Supplementary Table 1. Patients were divided into three groups based on melatonin concentration in their follicular fluid samples: Group $A ; \leq 30$ $\mathrm{pg} / \mathrm{mL}$, Group B; $>70$ to $\leq 110 \mathrm{pg} / \mathrm{mL}$ ), and Group C; $>111$ to $\leq 385 \mathrm{pg} / \mathrm{mL}$. Of the 425 oocytes with associated ff samples, 55 oocytes did not fertilize nor reach the 1PN stage. From the results of our study, it was evident that increased concentration of cfDNA is found in $\mathrm{ff}$ samples of group A (median, $95 \% \mathrm{Cl}$ : 
$2.01(1.66 ; 3.42)$ compared to groups B (median, 95\% Cl: $1.03(0.41 ; 1.98)$ and C (median, 95\% Cl: 0.86 $(0.11 ; 1.03 ; p<0.001)$. Subsequently, ROS, TBARS, and $8-0 H d G$ were significantly higher in group $A$ than that found in group $B$ and $C$ patients $(p<0.001)$. Similarly, we observed that patients exhibiting decreased intrafollicular melatonin concentration $(\leq 30 \mathrm{pg} / \mathrm{mL}$ ) have a significantly lower total antioxidant capacity [median, 95\% Cl: 238 (198; 305; Kruskal-Wallis test, $p<0.001$ ] (Table 1). However, we could not find any significant association among subgroups of patients regarding age, $\mathrm{BMI}$, and baseline endocrine parameters such as FSH, AMH, LH, and TSH levels. Additionally, we also screened for five selected microRNAs and detected miR-663b in 266/295 samples, miR-320a (284/295 samples), miR-766-3p (279/295 samples), miR-132-3p (268/295) and miR-16-5p in 273 out of 295 samples. 
Table 1

Relationship between different concentrations of melatonin and intrafollicular parameters

\begin{tabular}{|c|c|c|c|c|c|}
\hline Characteristics & $\begin{array}{l}\text { Group A (Intra- } \\
\text { follicular } \\
\text { Melatonin } \\
\text { Concentration } \\
\leq 30 \mathrm{pg} / \mathrm{ml} \text { ) }\end{array}$ & $\begin{array}{l}\text { Group B (Intra- } \\
\text { follicular } \\
\text { Melatonin } \\
\text { Concentration } \\
>70 \text { to } \leq 110 \\
\mathrm{pg} / \mathrm{ml} \text { ) }\end{array}$ & $\begin{array}{l}\text { Group C (Intra- } \\
\text { follicular } \\
\text { Melatonin } \\
\text { Concentration } \\
>111 \text { to } \leq 385 \\
\text { pg/ml) }\end{array}$ & $\begin{array}{l}\text { Kruskal- } \\
\text { Wallis } \\
\text { test }(P \\
\left.\text { value* }^{*}\right)\end{array}$ & $\begin{array}{l}P \\
\text { value** }\end{array}$ \\
\hline $\begin{array}{l}\text { Number of women } \\
{[n(\%)]}\end{array}$ & $97(32.8)$ & 145 (49.1) & $53(17.9)$ & - & - \\
\hline $\begin{array}{l}\text { Age (Years) [mean } \\
\pm \text { SD) }\end{array}$ & $33.85 \pm 2.11$ & $32.10 \pm 3.03$ & $30.14 \pm 4.41$ & 0.332 & - \\
\hline \multirow{2}{*}{$\begin{array}{l}\mathrm{BMI}\left(\mathrm{kg} / \mathrm{m}^{2}\right) \text { [mean } \\
\pm \mathrm{SD})[\text { median }(95 \% \\
\text { population limit)] }\end{array}$} & $28.52 \pm 0.99$ & $26.29 \pm 1.77$ & $25.89 \pm 2.25$ & \multirow[t]{2}{*}{0.455} & \multirow[t]{2}{*}{-} \\
\hline & $\begin{array}{l}22.33(19.8 \\
23.8)\end{array}$ & $\begin{array}{l}21.9(20.8 \\
23.8)\end{array}$ & $\begin{array}{l}22.01 \\
23.13)\end{array}$ & & \\
\hline \multicolumn{6}{|l|}{$\begin{array}{l}\text { Intrafollicular } \\
\text { parameters }\end{array}$} \\
\hline \multirow{2}{*}{$\begin{array}{l}\text { Intrafollicular E2 } \\
\text { level (ng/ml) [mean } \\
\pm \text { SD) [median }(95 \% \\
\text { population limit)] }\end{array}$} & $\begin{array}{l}411.2 \pm \\
113.69\end{array}$ & $\begin{array}{l}598.15 \pm \\
258.74\end{array}$ & \multirow{2}{*}{$\begin{array}{l}745.36 \pm 288.98 \\
698.3(497 \\
915.3)\end{array}$} & \multirow[t]{2}{*}{$<0.001$} & \multirow{2}{*}{$\begin{array}{l}<0.001^{a b}, \\
0.002^{c}\end{array}$} \\
\hline & $\begin{array}{l}389.3(295.7 \\
524.2)\end{array}$ & $\begin{array}{l}566.1(488.6 \\
830.1)\end{array}$ & & & \\
\hline \multirow{2}{*}{ 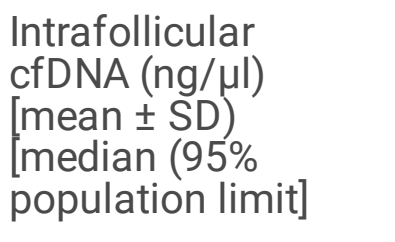 } & $2.97 \pm 1.36$ & $0.97 \pm 0.85$ & $0.81 \pm 0.52$ & \multirow[t]{2}{*}{$<0.001$} & \multirow{2}{*}{$<0.001^{a}$} \\
\hline & $\begin{array}{l}2.01(1.66 \\
3.42)\end{array}$ & $\begin{array}{l}1.03(0.41 ; \\
1.98)\end{array}$ & $0.86(0.11 ; 1.03)$ & & \\
\hline \multicolumn{6}{|l|}{$\begin{array}{l}\text { Intrafollicular } \\
\text { antioxidant status } \\
\text { and oxidative } \\
\text { Stress markers } \\
\text { [median (95\% } \\
\text { population limit] }\end{array}$} \\
\hline ROS (cpm) & $\begin{array}{l}38.98(25.8 \\
77.9)\end{array}$ & $\begin{array}{l}8.98(10.98 \\
15.09)\end{array}$ & $\begin{array}{l}6.89(3.09 \\
10.99)\end{array}$ & $<0.001$ & $\begin{array}{l}<0.001^{\mathrm{a}} \\
\mathrm{b}\end{array}$ \\
\hline TAC $(\mu \mathrm{M} / \mathrm{L})$ & $238(198 ; 305)$ & $965(698 ; 1067)$ & $\begin{array}{l}1275(883 ; \\
1765)\end{array}$ & $<0.001$ & $\begin{array}{l}<0.001^{\mathrm{a}} \\
\mathrm{b}, 0.003^{\mathrm{c}}\end{array}$ \\
\hline TBARS $(\mu \mathrm{M} / \mathrm{L})$ & $\begin{array}{l}1.98(1.45 \\
2.87)\end{array}$ & $\begin{array}{l}0.95(0.60 \\
1.02)\end{array}$ & $0.65(0.45 ; 0.87)$ & $<0.001$ & $\begin{array}{l}<0.001^{\mathrm{a}} \\
\mathrm{b}\end{array}$ \\
\hline 8-OHdG (ng/mL) & $\begin{array}{l}1.68(0.89 ; \\
2.12)\end{array}$ & $\begin{array}{l}0.97(0.76 ; \\
1.09)\end{array}$ & $0.71(0.43 ; 0.91)$ & $<0.001$ & $\begin{array}{l}<0.001^{\mathrm{a}} \\
\mathrm{b}\end{array}$ \\
\hline $\mathbf{N}^{\mathbf{d}}[\mathrm{n}(\%)]$ & & & & & \\
\hline
\end{tabular}




\begin{tabular}{|llllll|} 
miR-663b & $74(76.2)$ & $134(92.4)$ & $47(88.6)$ & - & - \\
\hline miR-320a & $83(85.5)$ & $141(97.2)$ & $51(96.2)$ & - & - \\
\hline miR-766-3p & $69(71.1)$ & $129(88.9)$ & $49(92.4)$ & - & - \\
\hline miR-132-3p & $62(63.9))$ & $135(93.1)$ & $50(94.3)$ & - & - \\
\hline miR-16-5p & $52(53.6)$ & $127(87.5)$ & $48(90.5)$ & - & - \\
\hline
\end{tabular}

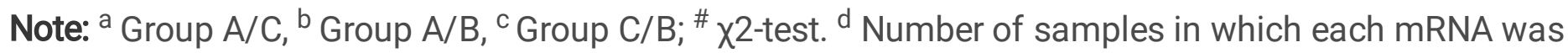
detected out of a total number of samples analyzed. E2, 17ß-estradiol; ROS, reactive oxygen species; TAC, total antioxidant capacity; LPO, lipid peroxidation; TBARS, Thiobarbituric acid reactive substances; 8OHdG, 8-hydroxy-2-deoxyguanosine. Intra-follicular data for patients are also subjected to statistical differences, $P^{\star}=$ Kruskal-Wallis test, $P^{\star \star}=$ pairwise comparisons between sub-groups, and $\mathrm{p}<0.05$ considered statistically significant.

\section{Intrafollicular melatonin concentration can predict IVF outcome parameters}

The characterization of IVF outcome parameters based on a pairwise comparison test between the groups is shown in Table 2. Patients with $\leq 30 \mathrm{pg} / \mathrm{mL}$ intrafollicular melatonin levels have significantly decreased IVF outcome parameters, particularly they have a low fertilization rate, a reduced number of mature oocytes, and high-quality day 3 embryos compared to other subgroups. Our results showed that IVF outcome parameters were better in group B and achieved a maximum in group C (Kruskal-Wallis test, $p<0.001)$ with the same rising tendency as the intrafollicular melatonin levels between the groups (Kruskal-Wallis test, $\mathrm{p}<0.001$ ). Correlation of intrafollicular melatonin concentration on IVF outcome parameters was given in supplementary Table 2, which shows that melatonin levels have a positive correlation with the number of MII- oocytes $\left(r_{s}=0.712 ; p<0.001\right)$, normal fertilized oocytes $\left(r_{s}=0.731\right.$; $p<0.002)$, early cleaved zygotes $\left(r_{s}=0.697 ; p<0.001\right)$, blastomeres (6-8 cells) with regular symmetry $\left(r_{s}=\right.$ $0.641 ; p<0.001)$, high-quality day 3 embryos $\left(r_{s}=0.745 ; p<0.003\right)$ and have a negative correlation with fragmentation rate $\left(r_{s}=-0.812 ;<0.001\right)$. 
Table 2

Association between intrafollicular melatonin levels and IVF outcome parameters.

\begin{tabular}{|c|c|c|c|c|c|}
\hline Characteristics & $\begin{array}{l}\text { Group A } \\
\text { (Intra- } \\
\text { follicular } \\
\text { Melatonin } \\
\text { Concentration } \\
\leq 30 \mathrm{pg} / \mathrm{ml} \text { ) }\end{array}$ & $\begin{array}{l}\text { Group B (Intra- } \\
\text { follicular } \\
\text { Melatonin } \\
\text { Concentration } \\
>70 \text { to } \leq 110 \\
\mathrm{pg} / \mathrm{ml} \text { ) }\end{array}$ & $\begin{array}{l}\text { Group C (Intra- } \\
\text { follicular } \\
\text { Melatonin } \\
\text { Concentration } \\
>111 \text { to } \leq 385 \\
\mathrm{pg} / \mathrm{ml} \text { ) }\end{array}$ & $\begin{array}{l}\text { Kruskal- } \\
\text { Wallis } \\
\text { test }(P \\
\text { value*) }\end{array}$ & $P$-value** \\
\hline \multirow{2}{*}{$\begin{array}{l}\text { No. of Follicles } \\
\text { with }>18 \mathrm{~mm} \text { size } \\
\text { [mean } \pm \text { SD) } \\
\text { [median ( } 95 \% \\
\text { population limit)] }\end{array}$} & $15.91 \pm 2.43$ & $16.18 \pm 2.12$ & $17.31 \pm 1.08$ & \multirow[t]{2}{*}{0.165} & \multirow[t]{2}{*}{ - } \\
\hline & $\begin{array}{l}13.2(12.11 \pm \\
17.12)\end{array}$ & $\begin{array}{l}13.5(8.9 ; \\
16.21)\end{array}$ & $14.1(9 ; 17)$ & & \\
\hline \multirow{2}{*}{$\begin{array}{l}\text { No. of Retrieved } \\
\text { oocytes [mean } \pm \\
\text { SD) [median } \\
\text { (95\% population } \\
\text { limit)] }\end{array}$} & $13.34 \pm 3.88$ & $14.09 \pm 2.90$ & $15.4 \pm 2.09$ & \multirow[t]{2}{*}{0.543} & \multirow[t]{2}{*}{ - } \\
\hline & $11.6(2 ; 14)$ & $12(9 ; 15)$ & $14(11 ; 16)$ & & \\
\hline \multirow{2}{*}{$\begin{array}{l}\text { No. of MII- } \\
\text { oOcytes [mean } \pm \\
\text { SD) [median } \\
\text { (95\% population } \\
\text { limit)] }\end{array}$} & $4.41 \pm 0.94$ & $10.12 \pm 1.11$ & $13.31 \pm 2.11$ & \multirow[t]{2}{*}{$<0.001$} & \multirow[t]{2}{*}{$<0.001^{a b}$} \\
\hline & $4.11(1-4.5)$ & $\begin{array}{l}9.83(6.89 \\
11.11)\end{array}$ & $\begin{array}{l}12.12(10.21 ; \\
15.12)\end{array}$ & & \\
\hline \multirow{2}{*}{$\begin{array}{l}\text { No. of Fertilized } \\
\text { oocytes [mean } \pm \\
\text { SD) [median } \\
\text { (95\% population } \\
\text { limit)] }\end{array}$} & $3.99 \pm 0.95$ & $9.98 \pm 2.12$ & $12.98 \pm 2.01$ & \multirow[t]{2}{*}{$<0.001$} & \multirow[t]{2}{*}{$<0.001^{a}, 0.003^{b}$} \\
\hline & $3.21(1 ; 3.5)$ & $\begin{array}{l}8.11(6.98 ; \\
10.87)\end{array}$ & $\begin{array}{l}11.91(9.07 ; \\
14.41)\end{array}$ & & \\
\hline \multirow{2}{*}{$\begin{array}{l}\text { No. of Normal } \\
\text { Fertilized oocytes } \\
\text { [mean } \pm \text { SD) } \\
\text { [median ( } 95 \% \\
\text { population limit)] }\end{array}$} & $3.16 \pm 0.34$ & $9.65 \pm 1.98$ & $12.39 \pm 1.88$ & \multirow[t]{2}{*}{$<0.001$} & \multirow[t]{2}{*}{$<0.001^{a b c}$} \\
\hline & $2.98(1 ; 3)$ & $\begin{array}{l}8.69 \text { (6.32; } \\
9.21)\end{array}$ & $\begin{array}{l}11.01 \text { (9.10; } \\
14.13)\end{array}$ & & \\
\hline \multirow{2}{*}{$\begin{array}{l}\text { No. of Early } \\
\text { cleaved zygotes } \\
\text { [mean } \pm \text { SD) } \\
\text { [median ( } 95 \% \\
\text { population limit)] }\end{array}$} & $2.10 \pm 0.54$ & $8.91 \pm 1.09$ & $11.59 \pm 1.87$ & \multirow[t]{2}{*}{$<0.001$} & \multirow[t]{2}{*}{$<0.001^{a b c}$} \\
\hline & $1.89(1 ; 2.8)$ & $\begin{array}{l}8.21 \text { (7.09; } \\
9.01)\end{array}$ & $\begin{array}{l}10.61(8.14 ; \\
12.58)\end{array}$ & & \\
\hline \multirow{2}{*}{$\begin{array}{l}\text { No. of } \\
\text { blastomeres (6-8 } \\
\text { cells) with } \\
\text { regular symmetry } \\
\text { at day } 3 \text { [mean } \pm \\
\text { SD) [median } \\
\text { (95\% population } \\
\text { limit)] }\end{array}$} & $2.01 \pm 0.12$ & $7.26 \pm 1.01$ & $10.84 \pm 1.88$ & \multirow[t]{2}{*}{$<0.001$} & \multirow[t]{2}{*}{$<0.001^{\mathrm{abc}}$} \\
\hline & $1.62(1 ; 2)$ & $\begin{array}{l}7.15(6.09 ; \\
8.81)\end{array}$ & $\begin{array}{l}10.2(9.18 \\
11.86)\end{array}$ & & \\
\hline \multirow{2}{*}{$\begin{array}{l}\text { No. of High- } \\
\text { Quality day } 3 \\
\text { embryos [mean } \pm \\
\text { SD) [median }\end{array}$} & $1.06 \pm 0.76$ & $6.32 \pm 1.43$ & $9.65 \pm 1.92$ & \multirow[t]{2}{*}{$<0.001$} & \multirow{2}{*}{$\begin{array}{l}<0.001^{\mathrm{a}} \\
0.004^{\mathrm{b}}\end{array}$} \\
\hline & $1(0 ; 2)$ & $\begin{array}{l}6.21(5.31 ; \\
6.81)\end{array}$ & $\begin{array}{l}7.94(6.98 ; \\
9.87)\end{array}$ & & \\
\hline
\end{tabular}


(95\% population

limit)]

$\begin{array}{llllll}\begin{array}{l}\text { No. of Embryos } \\ \text { suitable for }\end{array} & 1.03 \pm 0.65 & 6.22 \pm 0.98 & 9.53 \pm 1.68 & <0.001 & <0.001^{\text {abc }} \\ \text { transfer [mean } \pm & 1.01(1-2.5) & 6.18(4.87 ; & 7.68(7.09 ; & \\ \begin{array}{l}\text { SD) [median } \\ (95 \% \text { population }\end{array} & & 6.58) & 9.13) & \\ \text { limit)] } & & & & \\ \begin{array}{l}\text { Fragmentation } \\ \text { rate (\%) }\end{array} & 20-25 & 8-15 & \leq 10 & \end{array}$

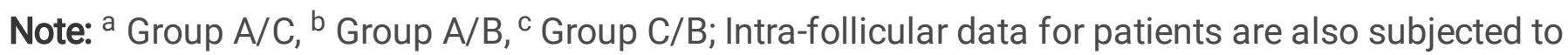
statistical differences, $\mathrm{P}^{*}=$ Kruskal-Wallis test, $\mathrm{P}^{\star \star}=$ pairwise comparisons between sub-groups and $p<0.05$ considered statistically significant.

\section{Intrafollicular cfDNA content and IVF outcome parameters among idiopathic patients}

The significant impact of intrafollicular melatonin concentration on cfDNA content of $f f$ is given in Table 3 , which shows that the higher level of intrafollicular melatonin significantly reduces the cfDNA content between groups. Among the analyzed idiopathic patients, no statistically significant differences have existed for the association of cfDNA concentration, and the number of oocytes retrieved, normally fertilized oocytes and early cleaved zygotes. However, when we compared the IVF outcome parameters such as number of MIl- oocytes, blastomeres with a regular symmetry, high-quality day 3 embryos and fragmentation rate, we observed that cfDNA content was significantly higher in group A than group B and $C(p<0.001)$ ( Table 3$)$. Studying the correlation between cfDNA levels and IVF outcome parameters shows that the highest cfDNA levels are related to the lowest IVF outcome parameters and decreased melatonin concentration (Supplementary Table 3 and Fig.1S). 
Table 3

Association between cfDNA concentration in follicular fluid of mature follicles and IVF laboratory parameters.

\begin{tabular}{|c|c|c|c|c|c|}
\hline Characteristics & $\begin{array}{l}\text { Intrafollicular } \\
\text { cfDNA } \\
\text { concentration } \\
\text { (ng/ } \mu \mathrm{l} \text { ) in } \\
\text { Group A }\end{array}$ & $\begin{array}{l}\text { Intrafollicular } \\
\text { cfDNA } \\
\text { concentration } \\
\text { (ng/ } / \text { l) in } \\
\text { Group B }\end{array}$ & $\begin{array}{l}\text { Intrafollicular } \\
\text { cfDNA } \\
\text { concentration } \\
\text { (ng/ } \mu \mathrm{l}) \text { in } \\
\text { Group C }\end{array}$ & $\begin{array}{l}\text { Kruskal- } \\
\text { Wallis } \\
\text { test }(P \\
\text { value*) }\end{array}$ & $P$-value** \\
\hline $\begin{array}{l}\text { Retrieved oocytes } \\
\text { (mean } \pm \text { SD) } \\
{[(95 \% \text { population }} \\
\text { limit)] }\end{array}$ & $\begin{array}{l}2.91 \pm 0.78 \\
{[1.5 ; 2.9]}\end{array}$ & $\begin{array}{l}2.53 \pm 0.43 \\
{[1.02 ; 2.78]}\end{array}$ & $\begin{array}{l}2.21 \pm 0.13 \\
{[1.01 ; 2.5]}\end{array}$ & 0.421 & - \\
\hline $\begin{array}{l}\text { MII- oocytes } \\
\text { (mean } \pm \text { SD) } \\
{[(95 \% \text { population }} \\
\text { limit)] }\end{array}$ & $\begin{array}{l}2.00 \pm 0.86 \\
{[0.98 ; 2.18]}\end{array}$ & $\begin{array}{l}1.64 \pm 0.97 \\
{[0.78 ; 1.93]}\end{array}$ & $\begin{array}{c}1.53 \pm 0.32 \\
{[0.98 ; 1.82]}\end{array}$ & $<0.001$ & $<0.001^{a}$ \\
\hline $\begin{array}{l}\text { Fertilized oocytes } \\
\text { (mean } \pm \text { SD) } \\
{[(95 \% \text { population }} \\
\text { limit)] }\end{array}$ & $\begin{array}{l}1.86 \pm 0.98 \\
{[1.21 ; 1.98]}\end{array}$ & $\begin{array}{l}1.51 \pm 0.65 \\
{[0.98 ; 1.80]}\end{array}$ & $\begin{array}{c}1.43 \pm 0.14 \\
{[0.91 ; 1.73]}\end{array}$ & 0.214 & - \\
\hline $\begin{array}{l}\text { Normal fertilized } \\
\text { oocytes (mean } \pm \\
\text { SD) }[(95 \% \\
\text { population limit)] }\end{array}$ & $\begin{array}{l}1.81 \pm 0.71 \\
{[1.14 ; 1.86]}\end{array}$ & $\begin{array}{l}1.48 \pm 0.88 \\
{[0.92 ; 1.91]}\end{array}$ & $\begin{array}{c}1.40 \pm 0.77 \\
{[0.81 ; 1.69]}\end{array}$ & 0.143 & - \\
\hline $\begin{array}{l}\text { Early cleaved } \\
\text { zygote (mean } \pm \\
\text { SD) }[(95 \% \\
\text { population limit)] }\end{array}$ & $\begin{array}{l}1.78 \pm 0.65 \\
{[1.08 ; 1.81]}\end{array}$ & $\begin{array}{l}1.32 \pm 0.54 \\
{[0.87 ; 1.53]}\end{array}$ & $\begin{array}{c}1.01 \pm 0.62 \\
{[0.93 ; 1.32]}\end{array}$ & 0.145 & - \\
\hline $\begin{array}{l}\text { Blastomeres (6-8 } \\
\text { cells) with regular } \\
\text { symmetry at day } 3 \\
\text { [mean } \pm \text { SD) [(95\% } \\
\text { population limit)] }\end{array}$ & $\begin{array}{l}1.98 \pm 0.54 \\
{[1.02 ; 2.31]}\end{array}$ & $\begin{array}{l}0.98 \pm 0.76 \\
{[0.19 ; 1.09)}\end{array}$ & $\begin{array}{l}0.51 \pm 0.98 \\
{[0.16 ; 0.99)}\end{array}$ & $<0.001$ & $<0.001^{\mathrm{a}}, 0.002^{\mathrm{b}}$ \\
\hline $\begin{array}{l}\text { High-Quality day } 3 \\
\text { embryos [mean } \pm \\
\text { SD) [median ( } 95 \% \\
\text { population limit)] }\end{array}$ & $\begin{array}{l}1.59 \pm 0.97 \\
{[1.04 ; 1.94]}\end{array}$ & $\begin{array}{l}0.88 \pm 0.12 \\
{[0.15 ; 1.13]}\end{array}$ & $\begin{array}{l}0.43 \pm 0.31 \\
{[0.49 ; 0.99]}\end{array}$ & $<0.001$ & $<0.001^{\mathrm{a}}, 0.01^{\mathrm{b}}$ \\
\hline $\begin{array}{l}\text { Embryos suitable } \\
\text { for transfer [mean } \\
\pm \text { SD })[(95 \% \\
\text { population limit)] }\end{array}$ & $\begin{array}{l}1.81 \pm 56 \\
{[1.12 ; 1.99]}\end{array}$ & $\begin{array}{l}1.46 \pm 0.49 \\
{[0.56 ; 1.01]}\end{array}$ & $\begin{array}{c}0.91 \pm 0.64 \\
{[0.71 ; 0.88]}\end{array}$ & 0.214 & - \\
\hline $\begin{array}{l}\text { Fragmentation } \\
\text { rate }(\leq 25 \%)\end{array}$ & $\begin{array}{l}1.91 \pm 0.76 \\
{[1.03 ; 1.96]}\end{array}$ & $\begin{array}{l}0.92 \pm 0.97 \\
{[0.17 ; 0.88]}\end{array}$ & $\begin{array}{l}0.49 \pm 0.29 \\
{[0.17 ; 0.53]}\end{array}$ & $<0.001$ & $<0.001^{a}, 0.003^{b}$ \\
\hline
\end{tabular}

Note: ${ }^{\text {a }}$ Group A/C, ${ }^{b}$ Group A/B, ${ }^{c}$ Group C/B; Group A (Intra-follicular Melatonin Concentration $\leq 30$ $\mathrm{pg} / \mathrm{ml}$ ), Group B (Intra-follicular Melatonin Concentration $>70$ to $\leq 110 \mathrm{pg} / \mathrm{ml}$ ), Group C (Intra-follicular 
Melatonin Concentration $>111$ to $\leq 385 \mathrm{pg} / \mathrm{ml}$ ). Intra-follicular data for patients are also subjected to statistical differences, $\mathrm{P}^{*}=$ Kruskal-Wallis test, $\mathrm{P}^{\star \star}=$ pairwise comparisons between sub-groups, and $p<0.05$ considered statistically significant.

\section{Effect of melatonin concentration on intrafollicular oxidative balance and day 3 embryo quality}

The association of intrafollicular melatonin levels and oxidative stress markers is shown in Supplementary Table 4. The comparison between different subgroups shows significant differences. Besides the expected differences regarding oxidative status, patients with lower melatonin concentration $(\leq 30 \mathrm{pg} / \mathrm{ml}$ ) have higher levels of oxidative stress markers and exhibited a higher number of impaired quality day 3 embryos than patients with moderate to high levels of melatonin. Of note, TAC levels have markedly increased in superior quality day 3 embryos [median (95\% Cl; $1164.3(877.45 ; 1467.75 ; p<0.001$ ] developed from oocytes containing higher levels of intrafollicular melatonin $(>111 \mathrm{pg} / \mathrm{ml})$. Likewise, they distinctively exhibit a positive correlation $\left(R^{2}=0.870 ; p<0.001\right)$ (Fig. $\left.2 S\right)$.

Table 4

Expression of miRNAs between the three groups.

\begin{tabular}{|c|c|c|c|c|c|c|c|c|c|}
\hline \multicolumn{4}{|c|}{$\begin{array}{l}\text { Group A (Intra-follicular Melatonin } \\
\text { Concentration } \leq 30 \mathrm{pg} / \mathrm{ml} \text { ) }\end{array}$} & \multicolumn{3}{|c|}{$\begin{array}{l}\text { Group B (Intra-follicular } \\
\text { Melatonin Concentration } \\
>70 \text { to } \leq 110 \mathrm{pg} / \mathrm{ml} \text { ) }\end{array}$} & \multicolumn{3}{|c|}{$\begin{array}{l}\text { Group C (Intra-follicular } \\
\text { Melatonin Concentration } \\
>111 \text { to } \leq 385 \mathrm{pg} / \mathrm{ml} \text { ) }\end{array}$} \\
\hline & $\begin{array}{l}\text { Raw } \\
\mathrm{Ct}\end{array}$ & $\Delta \mathrm{Ct}$ & RQ & Raw Ct & $\Delta \mathrm{Ct}$ & RQ & Raw Ct & $\Delta \mathrm{Ct}$ & $\mathrm{RQ}$ \\
\hline $\begin{array}{l}\text { miR- } \\
663 b\end{array}$ & 31.986 & 12.765 & 0.231 & 25.976 & 6.143 & 13.987 & 23.927 & 4.345 & 49.432 \\
\hline $\begin{array}{l}\text { miR- } \\
320 a\end{array}$ & 32.098 & 12.987 & 0.227 & 24.987 & 4.823 & 38.945 & 22.987 & 3.545 & 88.766 \\
\hline $\begin{array}{l}\text { miR- } \\
766- \\
3 p\end{array}$ & 33.987 & 13.098 & 0.113 & 25.156 & 5.980 & 10.765 & 24.183 & 4.839 & 38.098 \\
\hline $\begin{array}{l}\text { miR- } \\
132- \\
3 p\end{array}$ & 33.997 & 13.154 & 0.143 & 24.432 & 4.909 & 42.981 & 23.546 & 4.198 & 44.456 \\
\hline $\begin{array}{l}\text { miR- } \\
16-5 p\end{array}$ & 29.688 & 10.213 & 0.9754 & 26.098 & 7.654 & 5.443 & 25.872 & 5.587 & 24.098 \\
\hline
\end{tabular}

Note: Internal reference miR 16. $\Delta \mathrm{Ct}=$ Raw $\mathrm{Ct}$ target miRNA - Raw Ct miR-16. Inclusion criteria: high expression -level miRNAs with (Raw $\mathrm{Ct}<30$ and $\Delta \mathrm{Ct}(\mathrm{mRNA})<10)$. Low expression -level miRNAs with (Raw $\mathrm{Ct}>30$ and $\Delta \mathrm{Ct}(\mathrm{mRNA})>10)$. 


\section{Melatonin mediated miRNAs expression profile in follicular fluid samples is associated with IVF outcome parameters}

Under the influence of melatonin, miRNAs have shown different expression patterns that vary from group A to $C$ with the same trend as the intrafollicular melatonin levels differ among the groups (see Fig. 1). As shown in Table 4, the relative expression levels of miR-663b, miR-320a, miR-766-3p, miR-132-3p, and miR-16-5p were significantly upregulated in group $B$ and $C($ Raw $C t<30)$ compared to group $A(R a w ~ C t>$ 30). In group $A$ the relative expression levels of miR-766-3p and miR-132-3p were significantly reduced $(p<0.002)$ in comparison to group $B$ and $C$. While the relative expression of miR-766-3p and miR-132-3p in $\mathrm{ff}$ of groups $\mathrm{B}$ and $\mathrm{C}$ were slightly upregulated but did not reach the significant threshold (see Fig. 1). To examine the possible correlation between miRNAs in ff and day 3 embryo quality, we compared cases of high-quality embryos to impaired-quality embryos. The correlation analysis results were summed up in Supplementary Table 5, which shows a significant positive correlation between miRNAs' relative expression and day 3 embryo quality. In all studied subjects, when we compared the fold change ( 2 $\Delta \Delta \mathrm{Crt})$ of miRNAs in $\mathrm{ff}$ samples between high quality and impaired quality embryos on day 3 , we observed that miR-663b ( $F C=1.97, p=0.02)$, miR-320a $(F C=2.01, p=0.01)$, miR-766-3p $(F C=2.52, p=$ $0.03)$, miR-132-3p ( $F C=2.41, p=0.04)$ and miR-16-5p $(F C=1.89, p=0.05)$ exhibited significantly different expression levels respectively (see Table S5).

\section{Predictive model for high-quality embryo selection}

A predictive model for high-quality embryos is depicted in Fig. 2. Accordingly, the largest areas under the ROC were $0.89[95 \% \mathrm{Cl} ; 0.83 ; 0.96](p=0.001)$ at cut-off values of $\geq 0.79$ along with $75 \%$ sensitivity and $92.5 \%$ specificity for miR-320a and $0.88(0.82 ; 0.94)$, with $95 \%$ sensitivity and $72.5 \%$ specificity $(\mathrm{p}=$ 0.003 , at cut-off value $\geq 0.59$ ) for miR-132-3p (see Fig. $2 A$ ). These results showed that miR-320a predicts IVF outcome parameters better than miR-132-3p (see Table 5). Likewise, combination of all miRNAs did not improve the AUC of miR-320a (0.89) but enhance the senstivity to $87 \%$ and slightly decrease the specificity to $83 \%$ which may offer a potent non-invasive diagnostic tool in the selection of high-quality day 3 embryos (see Table 5). ROC analysis for oxidative stress markers showed that TAC have the highest AUC value 0.85 [95\% CL; 0.77; 0.93] with cut-off value of 0.89 and showed $90.2 \%$ sensitivity and $80.3 \%$ specificity $(\mathrm{p}=0.001)$. On the other hand ROS have lowest AUC value $0.65[95 \% \mathrm{Cl} ; 0.54 ; 0.76]$ at cut-off value of 1.88 exhibited $63.3 \%$ senstivity and $70 \%$ specificity $(p=0.021)$ (see Fig. 2B).

Furthermore, the AUC value for the combination of all evaluated stress markes was 0.78 with sensitivity of $83.2 \%$ and specificity of $78.3 \%(p=0.001)$ as given in (see Table 5$)$. For melatonin AUC value was 0.85 [cut-off points, $95 \% \mathrm{Cl}: 0.80,0.78 ; 0.92$ ] with sensitivity $90.7 \%$ and specificity $72.6 \%$ whereas cfDNA have AUC value 0.76 [cut-off points, $95 \% \mathrm{Cl}: 0.61,0.67 ; 0.85$ ] along with $82.4 \%$ senstivity and $66.7 \%$ specificity (see Fig. 2C). 
Table 5

Predictive values of sensitivity and specificity estimates for the probability of obtaining the best quality embryo develops from mature oocytes.

\begin{tabular}{|c|c|c|c|c|c|c|}
\hline & $\begin{array}{l}\text { AUC }[(95 \% \\
\text { population } \\
\text { limit })]\end{array}$ & S.E.M & $\begin{array}{l}* p \\
\text { Value }\end{array}$ & $\begin{array}{l}{ }^{*} \text { Cut- } \\
\text { off } \\
\text { point }\end{array}$ & Sensitivity & Specificity \\
\hline miR-663b & $\begin{array}{l}0.87(0.80 \\
0.94)\end{array}$ & 0.036 & 0.002 & $\geq_{0.64}$ & 88.3 & 80 \\
\hline miR-320a & $\begin{array}{l}0.89(0.83 ; \\
0.96)\end{array}$ & 0.032 & 0.001 & $\geq_{0.79}$ & 75.0 & 92.5 \\
\hline miR-766-3p & $\begin{array}{l}0.86(0.79 \\
0.94)\end{array}$ & 0.037 & 0.001 & $\geq_{0.57}$ & 93.3 & 72.5 \\
\hline miR-132-3p & $\begin{array}{l}0.88(0.82 ; \\
0.94)\end{array}$ & 0.032 & 0.003 & $\geq_{0.59}$ & 95 & 72.5 \\
\hline miR-16-5p & $\begin{array}{l}0.85(0.78 \\
0.93)\end{array}$ & 0.039 & 0.001 & $\geq_{0.68}$ & 88.8 & 80.5 \\
\hline $\begin{array}{l}\text { Combination of } \mathrm{ff} \text { miR-663, } \\
320 \mathrm{a}, 766-3 p, 132-3 p \text { and } \\
16-5 p\end{array}$ & $\begin{array}{l}0.89(0.71 ; \\
0.93)\end{array}$ & 0.034 & 0.001 & - & 87 & 83 \\
\hline ROS & $\begin{array}{l}0.65(0.54 ; \\
0.76)\end{array}$ & 0.055 & 0.021 & 1.88 & 63.3 & 70 \\
\hline TAC & $\begin{array}{l}0.85(0.77 ; \\
0.93)\end{array}$ & 0.049 & 0.001 & 0.89 & 90.2 & 80.3 \\
\hline LPO & $\begin{array}{l}0.83(0.75 \\
0.91)\end{array}$ & 0.042 & 0.001 & 0.78 & 91.7 & 72.5 \\
\hline TBARS & $\begin{array}{l}0.69(0.58 \\
0.80)\end{array}$ & 0.052 & 0.003 & 0.71 & 80 & 40 \\
\hline 8-OHdG & $\begin{array}{l}0.75(0.65 ; \\
0.85)\end{array}$ & 0.058 & 0.001 & 0.75 & 93.3 & 60.1 \\
\hline $\begin{array}{l}\text { Combination of } \mathrm{ff} \\
\text { evaluated oxidative stress } \\
\text { markers }\end{array}$ & $\begin{array}{l}0.78(0.69 \\
0.85)\end{array}$ & 0.045 & 0.001 & - & 83.2 & 78.36 \\
\hline cfDNA & $\begin{array}{l}0.76(0.67 ; \\
0.85)\end{array}$ & 0.047 & 0.001 & 0.61 & 82.4 & 66.7 \\
\hline Melatonin & $\begin{array}{l}0.85(0.78 \\
0.92)\end{array}$ & 0.037 & 0.001 & 0.80 & 90.7 & 72.6 \\
\hline
\end{tabular}

Note: ${ }^{*}$ The null hypothesis was true area $=0.5$, after the adjustment of a number of attempts and the number of embryos. P-values in bold letters considered statistically significant $p<0.05$.

** Estimated cut points that maximize sensitivity and specificity for observed range predictors. 


\section{Multitargeting activity of melatonin upregulated miRNAs}

MicroRNAs are the genetic switches that fine-tune essential cellular responses and are required to streamline the signal transductions in several cell types. They are depicted as multivalent with single miRNA able to target numerous genes, thus regulating structural and functional molecules' expression within a pathway. In the present study, we identified several pathways that involve at least one of the studied miRNAs, including cytoskeletal organization, post-translational protein modification, cell cycle, oocyte meiosis, p53 signaling pathway, TGF-beta signaling pathway, and estrogen signaling pathway (see Fig. 3 and 3S).

\section{Discussion}

The intrafollicular microenvironment is a highly complex and critical indicator of an individual oocyte's developmental capability to be fertilized and mature into a good quality embryo. Primarily melatonin's positive impact on the intrafollicular microenvironment has been reported through melatonin-mediated gene expression [18]. This is the first attempt in unexplained infertile patients to establish a direct association between melatonin's antioxidant property and its impact on oocyte quality, fertilization rates, and embryo quality by exploring the relative expression patterns of five miRNAs and levels of cfDNA.

Our results established that patients with higher melatonin concentration were associated with elevated $17 \beta$-estradiol $\left(E_{2}\right)$ levels in their ff samples. $E_{2}$ is a key player in the final steps of oocyte's nuclear and cytoplasmic maturation [19]. The non-human primate study demonstrated that it can improve oocytes' developmental competence during in vitro maturation (IVM) [20]. Our study showed that patients with lower intrafollicular melatonin concentration exhibited a considerable oxidative imbalance in their $\mathrm{ff}$ (e.g., lower TAC levels and higher ROS, 8-OHdG, and TBARS levels), which jeopardizes the quality of oocytes and thus, hampers the oocyte's maturation along with limiting IVF outcome parameters. These findings follow much earlier research reporting that an imbalance between lipid peroxidation marker (TBARS) and antioxidant system plays a significant role in the pathogenesis of unexplained infertility [21]. Our study's outcome agrees with Jana et al, which revealed a direct relationship between low TAC levels and poor embryo quality and a sharp decline in fertilization rate [22]. Additionally, recent studies validated that decreased melatonin concentration is responsible for reduced TAC levels in the ff samples [23, 24]. These studies suggest that elevated follicular lipid peroxidation and lower TAC levels have a negative impact on IVF outcomes.

Over the past decade, advances in scientific knowledge have established that depreciation in intrafollicular melatonin assets resulted in excessive ROS production, which is responsible for single or double-strand DNA breaks, thus introducing mutations in nuclear DNA and reduces in mitochondrial function [25]. Together with this reference, our study tends to confirm that the concentration of ROS 
raised high in those patients who had lower levels of melatonin $(\leq 30 \mathrm{pg} / \mathrm{mL})$ than those who had moderate to higher concentrations in their ff samples. ROS production might be a result of suboptimal intrafollicular microenvironment or impaired metabolism of the developing oocyte. Increasing evidence highlights that melatonin is responsible for the upregulation of specific miRNAs, which control antioxidative enzymes' expression, more likely by acting as a potent free radical scavenger [26]. Similarly, another group reported that melatonin significantly upregulates genes' expression and the formation of proteins responsible for synthesizing antioxidant enzymes. Therefore, compromised antioxidant capacity leads to oxidative stress and flare-up the process of apoptosis [27].

One of the significant obstacles for IVF is oxidative damage to nuclear DNA that can be estimated by assessing intrafollicular cfDNA concentration and levels of 8-OHdG. The increased concentrations of cfDNA and 8-OHdG levels also indicate the embryo's inappropriate quality and often have a low success rate of pregnancy $[9,28]$. In the same context, our results showed that intrafollicular cfDNA concentration and 8-OHdG levels were significantly raised in good quality embryos of those patients who have decreased intrafollicular melatonin concentration $(\leq 30 \mathrm{pg} / \mathrm{mL})$. Alternatively, those patients who exhibited a higher concentration of melatonin $(>111 \mathrm{pg} / \mathrm{mL})$ in their $\mathrm{ff}$ samples have decreased intrafollicular cfDNA concentration and levels of 8-OHdG, which is in agreement with previous observations [29, 30]. Furthermore, in the present study, higher intrafollicular melatonin resulted in an enrichment of high-quality day 3 embryos leading to an increase in the availability of embryos suitable for transfer, ultimately resulting in an augmented future pregnancy rate per embryo transfer [31].

Our exploratory analysis exhibited that miRNAs such as miR-320a, miR766-3p, miR-132-3p, miR-16-5p, and miR-663b were significantly decreasing their relative expression in ff samples that have a lower concentration of intrafollicular melatonin $(\leq 30 \mathrm{pg} / \mathrm{mL})$ and yielded a smaller number of high-quality embryos on day 3. In agreement with our study, Feng et al compared miRNAs from the ff that generate poor-quality and top-quality embryos. They found that miR-132-3p and miR-16-5p were downregulated in the ff containing mature oocytes that produce a higher number of poor-quality embryos than top quality embryos and vice-versa [12,32]. However, the findings were statistically insignificant, and the effect of intrafollicular melatonin to regulate these miRNAs is mostly unknown. Bioinformatic analysis of studied miRNAs reveals a fundamental role in mediating genes that regulate vital aspects of multiple biological functions. They mediate cell-to-cell communication and target genes associated with follicular development, growth, and oocyte maturation, further indicating that these endogenous messengers have a critical role in oogenesis [33]. Subsequently, the molecular signature based on these miRNAs' differential levels enabled them to participate in the cell junction assembly, TGF-beta signaling, MAPK signaling, Wnt signaling PI3K-Akt pathway, Notch signaling, Estrogen signaling, and Hippo signaling pathways [34, 35].

Our study showed that miR-320 was among the highest expressed miRNA in ff samples, which have intrafollicular melatonin concentration $>111 \mathrm{pg} / \mathrm{mL}$. Similarly, Diez-frail et al observed that miR-320 was over the top-ten highest expressed miRNA in ff samples of good quality embryos [36]. Further, they reported that knockdown of miR-320a expression in mouse metaphase-Il oocytes resulting in embryos 
arrested at first cleavage. While very few can develop into top-quality embryos, indicating that the miR$320 \mathrm{a}$ has a potential role in modulating gene expression and regulating embryonic development [37]. Intriguingly, another investigation did not find miR-320 in ff [38]. This inconsistency in the results may be explained by genetic heterogeneity due to the population's different ethnic origins, resulting in varied gene expression in body fluids. Moreover, different stimulation protocols may be responsible for a varied expression of genes or miRNAs. Unsurprisingly, studies focused on the relationship between miR-320a relative expression levels in $\mathrm{ff}$ and embryonic development provides additional support to physiological and molecular mechanisms underlying in-vivo and in-vitro fertilization [32, 39, 40]. Evidence from another investigation has indicated that melatonin exerts its antioxidant activities by coordinating crosstalk between miRNAs and interrelated pathways [12].

Additionally, a transcriptome analysis reveals that high quality human preimplantation embryos secrete miR-320a that regulates the decidualized human endometrial stromal cells (hESCs) migration by targeting cell adhesion and cytoskeleton organization [32]. The outcome of their study specifies the promising effect of miR-320a to boost success rates in assisted reproduction. Previously, another piece of evidence indicates that miR766-3p, miR-132-3p, miR-16-5p, and miR-663b have decreased relative expression in the ff samples, yielding impaired quality embryos when compared with the top-quality embryos on day 3 [11]; this is in line with our findings. In the same frame of reference, a recent study conducted by Ragusa et al. confirmed the hypothesis that miR766-3p fine-tunes cellular responses, especially in the control of the cell cycle, play a vital role in the first phase of embryogenesis [41]. Conversely, Fu et al confirmed that miR-663b has a substantial negative correlation in $\mathrm{ff}$ of oocytes that produce viable blastocyst than those yielding poor-quality blastocyst [42]. However, the authors of the study did not mention the exact cause of infertility in the subjects. We speculated that it is potentially resulting from vast differences in patiens' selection, detection methodology and biological variability.

This study still holds some significant limitations, as other confounding factors were not addressed. First, the oocyte and embryo quality are associated with morphological scores and the number and composition of chromosomes; therefore, it is imperative to find the numerical chromosome euploidy by Preimplantation Genetic Diagnosis (PGD). Second, we were not able to knockdown miRNAs to assess their actual effects on embryonic development. Third, the primary goal is embryo quality, not live birth. Further studies should be planned to find a relationship between these miRNAs' expression in $\mathrm{ff}$ and chromosomal anomalies.

\section{Conclusion}

Conclusively, our study showed that melatonin antioxidant capability significantly impacts cfDNA concentration and specific miRNAs relative expression profile in the follicular ambient microenvironment on oocyte development and embryo quality. Therefore, it may be a potent non-invasive diagnostic tool to select high-quality day 3 embryos in the future with such promise. Furthermore, information compiled herein will improve our understanding that physiology of reproduction may categorize these miRNAs as non-invasive biomarkers. These unique miRNAs signature will enhance the quality of embryo selection 
and minimize the chance of multiple gestations, thus, eventually, improve the probability of successful IVF pregnancies. More comprehensive studies should be conducted on a genome-wide scale that must confirm melatonin's effect in the follicular microenvironment to regulate miRNA expression profiles related to oocyte maturation and embryo quality.

\section{Abbreviations}

IVF: In vitro fertilization, AFC: Antral follicle count, AMH anti-Müllerian hormone, PCOS: Polycystic Ovarian Syndrome, cfDNA: Cell-free DNA, ff: Follicular fluid. PGD: preimplantation genetic diagnosis, hESCs: human endometrial stromal cells, TAC: total antioxidant capacity, ROS: reactive oxygen species, SOD: superoxide dismutase, GSH: glutathione, miRNA: microRNA, ROC: receiving operating characteristics, $\mathrm{E}_{2}$ : $17 \beta$-estradiol BMl: body mass index, FSH: follicular stimulating hormone, LH: luteinizing hormone (LH), TSH: thyroid-stimulating hormone, TVS: transvaginal ultrasonography, COS: controlled ovarian stimulation, ICSI: intracytoplasmic sperm injection, TBARS: Thiobarbituric acid reactive substances, 8OHdG: 8-hydroxy-2'-deoxyguanosine, $\mathrm{Cl}$ : confidence interval.

\section{Declarations}

\section{Acknowledgments:}

We acknowledge the research initiative and gratefully thank Professor, Dr. RLK, Professor of Emeritus in Obstetrics and Gynecology, in manuscript editing. The study is supported by the infertility research center of HLA hospital.

\section{Funding:}

The infertility research center of HLA hospital

\section{Availability of data and materials:}

The data set used and analyzed during the current study is available from the corresponding author on reasonable request.

\section{Authors' Contributions:}

HLK, YLK Reviewing and Editing, Software; SB Conceptualization, Methodology, Software, Data curation, Project administration, Writing- Original draft preparation. Supervision, Writing- Reviewing and Editing; SA Formal analysis, Validation, Methodology, Investigation, Writing- Reviewing and Editing; CK Writing- 
Reviewing and Editing; SQ Data curation, Writing- Original draft preparation; ZH Reviewing and Editing, Methodology, Software NZT Writing- Reviewing and Editing; HHY Software, Validation, Methodology.

\section{Ethics approval and consent to participate:}

The study was approved by our Institutional Ethical Committee (IEC). Informed consent was obtained from all subjects before the research and publishing of the results of the investigation.

\section{Consent for Publication:}

Not applicable

\section{Competing interests:}

The authors declared that they have no conflict of interest.

\section{References}

1. Slominski AT, Hardeland R, Zmijewski MA, Slominski RM, Reiter RJ, Paus R: Melatonin: a cutaneous perspective on its production, metabolism, and functions. Journal of Investigative Dermatology 2018, 138(3):490-499.

2. Reiter RJ, Rosales-Corral SA, Manchester LC, Tan D-X: Peripheral reproductive organ health and melatonin: ready for prime time. International journal of molecular sciences 2013, 14(4):7231-7272.

3. Agarwal A, Sharma R, Gupta S, Harlev A, Ahmad G, Du Plessis SS, Esteves SC, Wang SM, Durairajanayagam $D$ : Oxidative stress in human reproduction: shedding light on a complicated phenomenon: Springer; 2017.

4. Kala M, Shaikh MV, Nivsarkar M: Equilibrium between anti-oxidants and reactive oxygen species: a requisite for oocyte development and maturation. Reproductive medicine and biology 2017, 16(1):2835.

5. Lu J, Wang Z, Cao J, Chen Y, Dong Y: A novel and compact review on the role of oxidative stress in female reproduction. Reproductive Biology and Endocrinology 2018, 16(1):80.

6. Cadet J, Davies KJ: Oxidative DNA damage \& repair: an introduction. Free Radical Biology and Medicine 2017, 107:2-12.

7. Pauli A, Rinn JL, Schier AF: Non-coding RNAs as regulators of embryogenesis. Nature Reviews Genetics 2011, 12(2):136-149.

8. Bouckenheimer J, Fauque P, Lecellier C-H, Bruno C, Commes T, Lemaître J-M, De Vos J, Assou S: Differential long non-coding RNA expression profiles in human oocytes and cumulus cells. Scientific reports 2018, 8(1):1-13. 
9. Khan HL, Bhatti S, Khan YL, Abbas S, Munir Z, Sherwani IARK, Suhail S, Hassan Z, Aydin HH: Cell-free nucleic acids and melatonin levels in human follicular fluid predict embryo quality in patients undergoing in-vitro fertilization treatment. Journal of Gynecology Obstetrics and Human Reproduction 2020, 49(1):101624.

10. Tanabe M, Tamura H, Taketani T, Okada M, Lee L, Tamura I, Maekawa R, Asada H, Yamagata Y, Sugino N: Melatonin protects the integrity of granulosa cells by reducing oxidative stress in nuclei, mitochondria, and plasma membranes in mice. Journal of Reproduction and Development 2014.

11. Machtinger R, Rodosthenous RS, Adir M, Mansour A, Racowsky C, Baccarelli AA, Hauser R:

Extracellular microRNAs in follicular fluid and their potential association with oocyte fertilization and embryo quality: an exploratory study. Journal of assisted reproduction and genetics 2017, 34(4):525533.

12. Feng R, Sang Q, Zhu Y, Fu W, Liu M, Xu Y, Shi H, Xu Y, Qu R, Chai R: MiRNA-320 in the human follicular fluid is associated with embryo quality in vivo and affects mouse embryonic development in vitro. Scientific reports 2015, 5:8689.

13. Lazzaroni-Tealdi E, Barad DH, Albertini DF, Yu Y, Kushnir VA, Russell H, Wu Y-G, Gleicher N: Oocyte scoring enhances embryo-scoring in predicting pregnancy chances with IVF where it counts most. PloS one 2015, 10(12):e0143632.

14. Dimopoulou M, Anifandis G, Messini C, Dafopoulos K, Kouris S, Sotiriou S, Satra M, Vamvakopoulos N, Messinis I: Follicular fluid oocyte/cumulus-free DNA concentrations as a potential biomolecular marker of embryo quality and IVF outcome. BioMed research international 2014, 2014.

15. Munakata $Y$, Shirasuna K, Kuwayama T, Iwata H: Cell-free DNA in medium is associated with the maturation ability of in vitro cultured oocytes. Journal of Reproduction and Development 2019:20182123.

16. Attaran M, Pasqualotto E, Falcone T, Goldberg JM, Miller KF, Agarwal A, Sharma RK: The effect of follicular fluid reactive oxygen species on the outcome of in vitro fertilization. International journal of fertility and women's medicine 2000, 45(5):314-320.

17. Diplock A, Symons MC, Rice-Evans C: Techniques in free radical research: Elsevier; 1991.

18. Tong J, Sheng S, Sun Y, Li H, Li W-P, Zhang C, Chen Z-J: Melatonin levels in follicular fluid as markers for IVF outcomes and predicting ovarian reserve. Reproduction (Cambridge, England) 2017, 153(4):443-451.

19. Sarhan D, El Mazny A, Taha T, Aziz A, Azmy O, Fakhry D, Torky H: Estradiol and luteinizing hormone concentrations in the follicular aspirate during ovum pickup as predictors of in vitro fertilization (IVF) outcome. Middle East Fertility Society Journal 2017, 22(1):27-32.

20. Zheng P, Si W, Bavister BD, Yang J, Ding C, Ji W: 17ß-Estradiol and progesterone improve in-vitro cytoplasmic maturation of oocytes from unstimulated prepubertal and adult rhesus monkeys. Human Reproduction 2003, 18(10):2137-2144.

21. Polak G, Rola R, Gogacz M, Kozioł-Montewka M, Kotarski J: Malonyldialdehyde and total antioxidant status in the peritoneal fluid of infertile women. Ginekologia polska 1999, 70(3):135-140. 
22. Jana SK, Babu N, Chattopadhyay R, Chakravarty B, Chaudhury K: Upper control limit of reactive oxygen species in follicular fluid beyond which viable embryo formation is not favorable. Reproductive Toxicology 2010, 29(4):447-451.

23. Espino J, Macedo M, Lozano G, Ortiz Á, Rodríguez C, Rodríguez AB, Bejarano I: Impact of melatonin supplementation in women with unexplained infertility undergoing fertility treatment. Antioxidants 2019, 8(9):338.

24. Fernando $S$, Osianlis T, Vollenhoven B, Wallace E, Rombauts L: A pilot double-blind randomised placebo-controlled dose-response trial assessing the effects of melatonin on infertility treatment (MIART): study protocol. BMJ open 2014, 4(8).

25. Ménézo $Y$, Dale $B$, Cohen M: DNA damage and repair in human oocytes and embryos: a review. Zygote 2010, 18(4):357-365.

26. Antolín I, Rodríguez C, Sáinz RM, Mayo JC, Uría H, Kotler ML, Rodríguez-Colunga MJ, Tolivia D, Menéndez-Peláez A: Neurohormone melatonin prevents cell damage: effect on gene expression for antioxidant enzymes. The FASEB journal 1996, 10(8):882-890.

27. Mayo J, Sainz R, Antolin I, Herrera F, Martin V, Rodriguez C: Melatonin regulation of antioxidant enzyme gene expression. Cellular and Molecular Life Sciences CMLS 2002, 59(10):1706-1713.

28. Seino T, Saito H, Kaneko T, Takahashi T, Kawachiya S, Kurachi H: Eight-hydroxy-2'-deoxyguanosine in granulosa cells is correlated with the quality of oocytes and embryos in an in vitro fertilizationembryo transfer program. Fertility and sterility 2002, 77(6):1184-1190.

29. Xu G, Zhao J, Liu H, Wang J, Lu W: Melatonin inhibits apoptosis and oxidative stress of mouse leydig cells via a SIRT1-dependent mechanism. Molecules 2019, 24(17):3084.

30. Liu R, Fu A, Hoffman AE, Zheng T, Zhu Y: Melatonin enhances DNA repair capacity possibly by affecting genes involved in DNA damage responsive pathways. BMC cell biology 2013, 14(1):1-8.

31. Tamura H, Takasaki A, Miwa I, Taniguchi K, Maekawa R, Asada H, Taketani T, Matsuoka A, Yamagata $\mathrm{Y}$, Shimamura K: Oxidative stress impairs oocyte quality and melatonin protects oocytes from free radical damage and improves fertilization rate. Journal of pineal research 2008, 44(3):280-287.

32. Berkhout RP, Keijser R, Repping S, Lambalk CB, Afınk GB, Mastenbroek S, Hamer G: High-quality human preimplantation embryos stimulate endometrial stromal cell migration via secretion of microRNA hsa-miR-320a. bioRxiv 2020.

33. Cargnello M, Roux PP: Activation and function of the MAPKs and their substrates, the MAPKactivated protein kinases. Microbiology and molecular biology reviews 2011, 75(1):50-83.

34. Wang D, Luo Y, Wang G, Yang Q: Circular RNA expression profiles and bioinformatics analysis in ovarian endometriosis. Molecular genetics \& genomic medicine 2019, 7(7):e00756.

35. Su S-C, Reiter RJ, Hsiao H-Y, Chung W-H, Yang S-F: Functional interaction between melatonin signaling and noncoding RNAs. Trends in Endocrinology \& Metabolism 2018, 29(6):435-445.

36. Diez-Fraile A, Lammens T, Tilleman K, Witkowski W, Verhasselt B, De Sutter P, Benoit Y, Espeel M, D'Herde K: Age-associated differential microRNA levels in human follicular fluid reveal pathways potentially determining fertility and success of in vitro fertilization. Human fertility 2014, 17(2):90-98. 
37. Salas-Huetos A, James ER, Aston KI, Jenkins TG, Carrell DT, Yeste M: The expression of miRNAs in human ovaries, oocytes, extracellular vesicles, and early embryos: a Systematic review. Cells 2019, 8(12):1564.

38. Santonocito M, Vento M, Guglielmino MR, Battaglia R, Wahlgren J, Ragusa M, Barbagallo D, Borzì P, Rizzari S, Maugeri M: Molecular characterization of exosomes and their microRNA cargo in human follicular fluid: bioinformatic analysis reveals that exosomal microRNAs control pathways involved in follicular maturation. Fertility and sterility 2014, 102(6):1751-1761. e1751.

39. Liang L-F, Qi S-T, Xian Y-X, Huang L, Sun X-F, Wang W-H: Protective effect of antioxidants on the prematuration aging of mouse oocytes. Scientific Reports 2017, 7(1):1-10.

40. Reza AMMT, Choi YJ, Han SG, Song H, Park C, Hong K, Kim JH: Roles of microRNAs in mammalian reproduction: from the commitment of germ cells to peri-implantation embryos. Biological Reviews 2019, 94(2):415-438.

41. Ragusa M, Barbagallo D, Chioccarelli T, Manfrevola F, Cobellis G, Di Pietro C, Brex D, Battaglia R, Fasano S, Ferraro B: CircNAPEPLD is expressed in human and murine spermatozoa and physically interacts with oocyte miRNAs. RNA biology 2019, 16(9):1237-1248.

42. Fu J, Qu R-g, Zhang Y-j, Gu R-h, Li X, Sun Y-j, Wang L, Sang Q, Sun X-x: Screening of miRNAs in human follicular fluid reveals an inverse relationship between microRNA-663b expression and blastocyst formation. Reproductive BioMedicine Online 2018, 37(1):25-32.

\section{Figures}


A

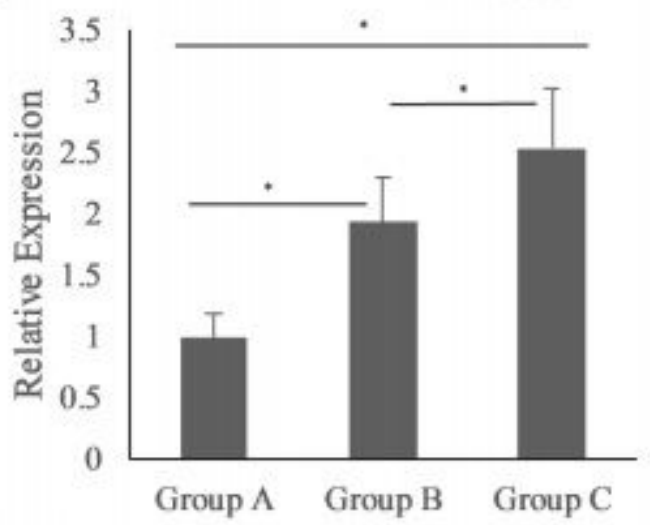

C

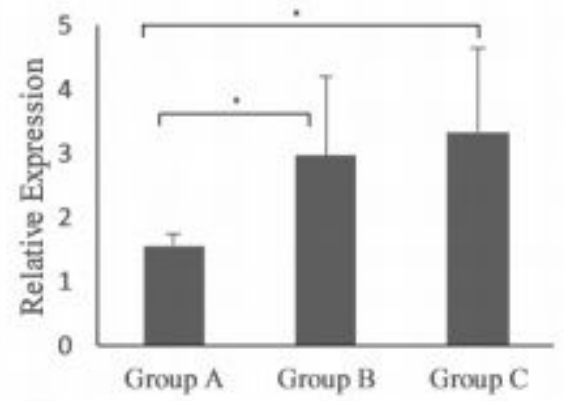

E
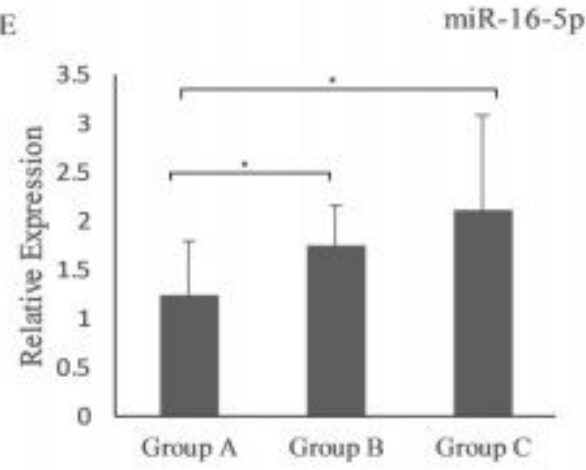

B miR-320a

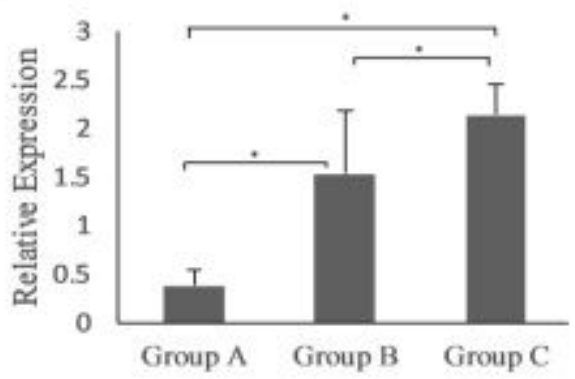

D

miR-132-3p

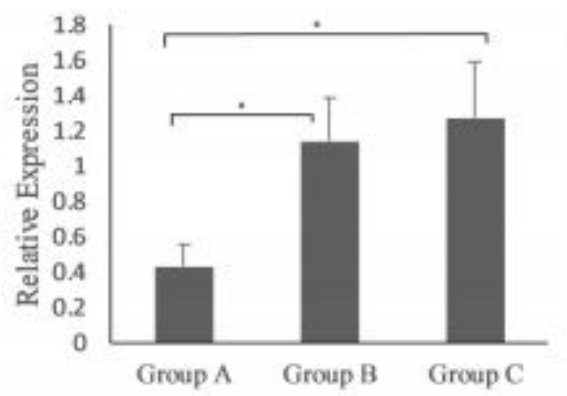

Figure 1

Comparison of miR-663b, miR-320a, miR-766-3p, miR-132-3p, and miR-16-5p expression levels relative to the concentration of intrafollicular melatonin. $\left(^{*}\right)$ Values indicate significant differences within the expression level of each miRNAs $(p<0.001)$. 

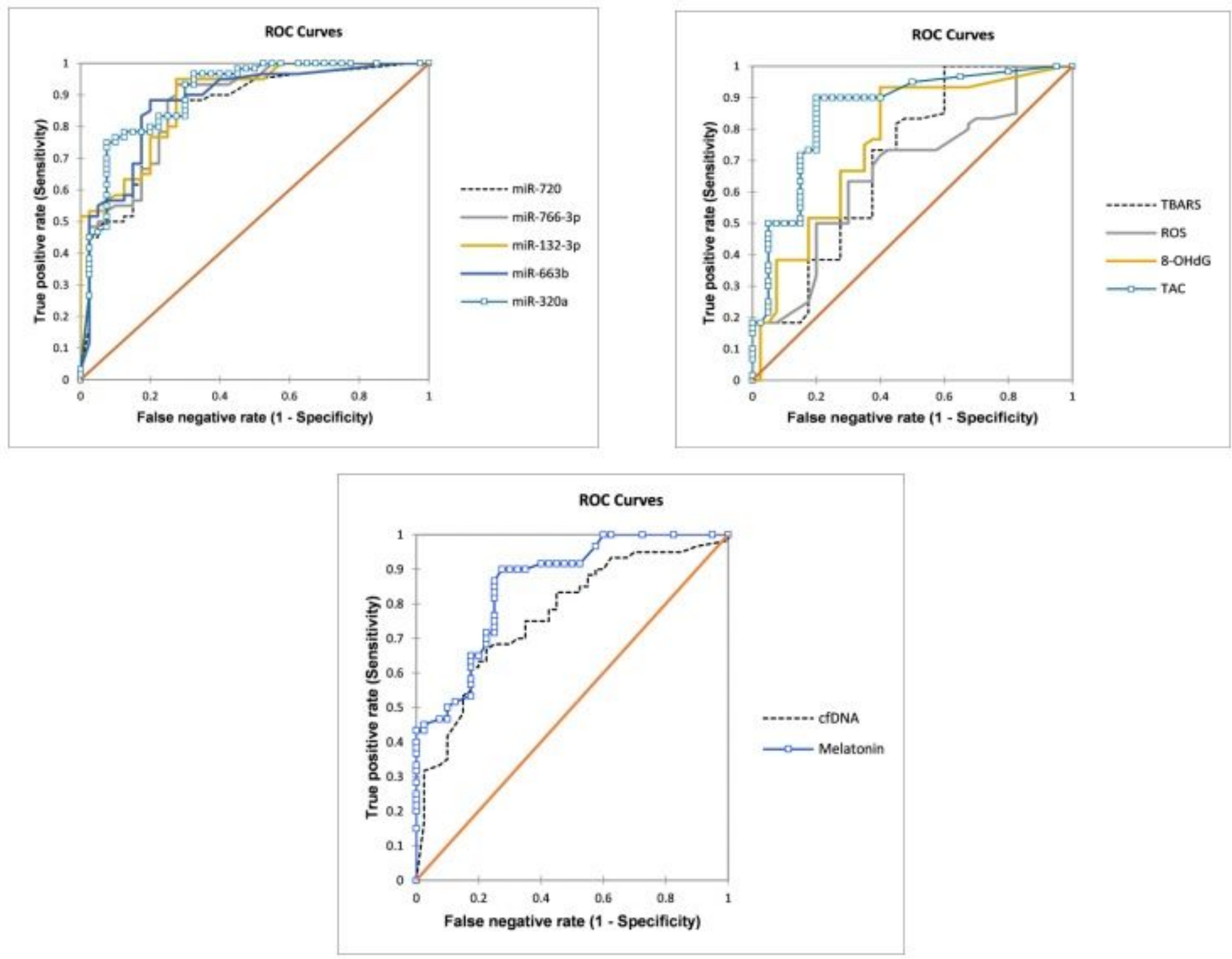

\section{Figure 2}

A predictive model for high-quality embryos selection based on melatonin concentration, cfDNA levels, oxidative stress markers, and expression patterns of miRNAs in ff samples of unexplained infertile patients. (A) ROC curve analysis to estimate the discriminative significance of ff concentrations of miR$663 b$, miR-320a, miR-766-3p, miR-132-3p, and miR-16-5p for prediction of good quality embryos. (B) ROC curve analysis to evaluate oxidative stress markers predictive values for good quality embryos selection. (C) ROC curve analysis assesses the predictive values of melatonin concentration and cfDNA levels for good quality embryo selection. 

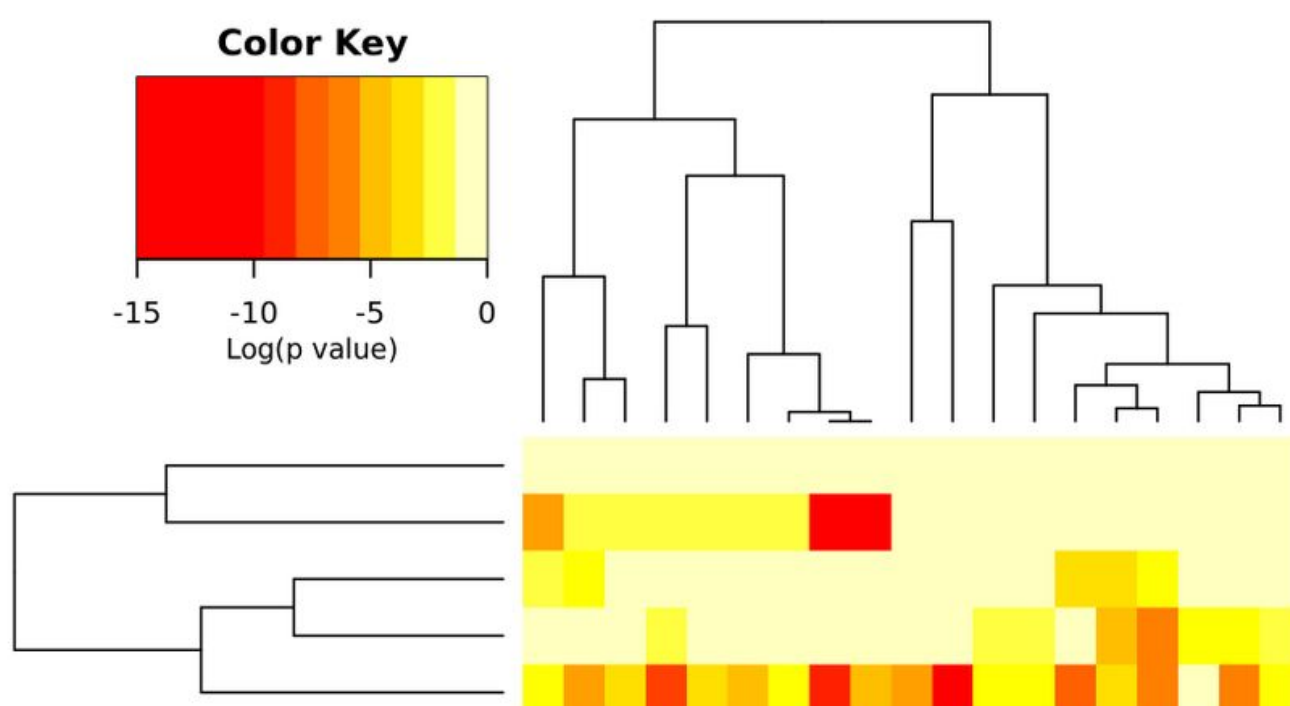

hsa-miR-663b|Tarbase

hsa-miR-766-3p|Tarbase

hsa-miR-132-3p|Tarbase

hsa-miR-320a|Tarbase

hsa-miR-16-5p|Tarbase

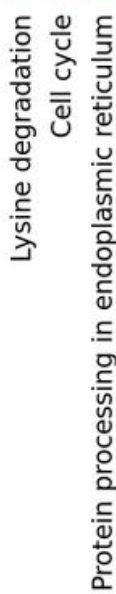

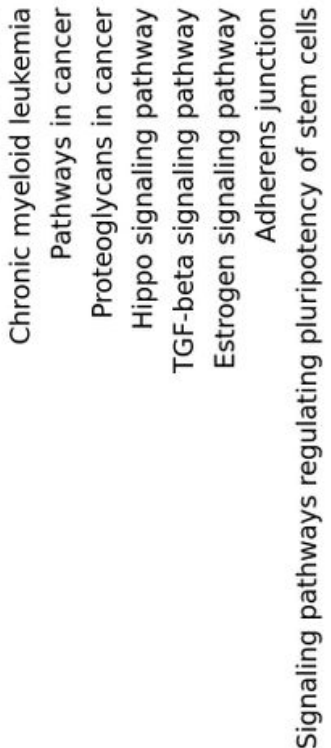

\section{Figure 3}

MiRNAs and predictive pathways heat map. Red color indicates high expression and lower $p$ values. Yellow color indicates intermediate expression.

\section{Supplementary Files}

This is a list of supplementary files associated with this preprint. Click to download.

- Figure.3S.png

- Figure.2SD.png

- Figure.2SC.png

- Figure.2SB.png 
- Figure.2SA.png

- Figure.1S.png

- Supplementytables.docx 